\title{
Polymeric Membrane Fluoride-Selective Electrodes Using Lewis Acidic Organo-Antimony(V) Compounds as lonophores
}

\author{
Long Li,* Yihao Zhang, Ying Li, Yinghui Duan, Yi Qian, Peidong Zhang, Qingjie Guo, and Jiawang Ding
}

Cite This: ACS Sens. 2020, 5, 3465-3473

Read Online
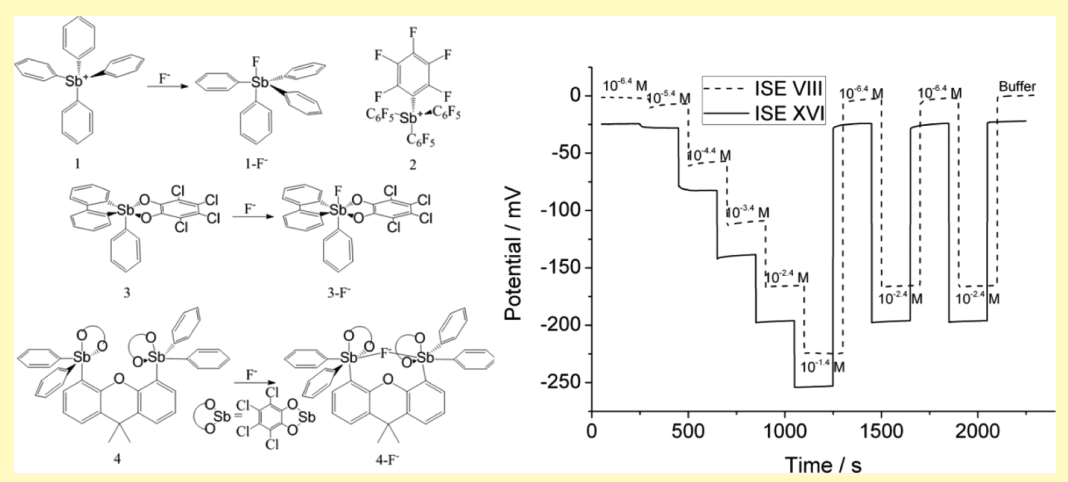

ABSTRACT: Four Lewis acidic organo-antimony $(\mathrm{V})$ compounds with strong binding affinity to fluoride were used for the first time as ionophores to fabricate polymeric membrane fluoride-selective electrodes. Improved detection limits and significant antiHofmeister selectivity could be achieved by optimizing ionophores, lipophilic additives, and plasticizers. Membrane electrodes fabricated with tetrakis-(pentafluorophenyl)stibonium (ionophore 2) performed best in detection limit, sensitivity, and selectivity. Optimal performance was obtained by fluoride with a slope of $-59.5 \mathrm{mV} /$ decade in the linear range of $1 \times 10^{-5}$ to $4 \times 10^{-2} \mathrm{M}$ and a detection limit of $5 \times 10^{-6} \mathrm{M}$. Studies on the influence of sample solution $\mathrm{pH}$ demonstrate that the best $\mathrm{pH}$ for fluoride determination is $\mathrm{pH}$ 3.0. All of the electrodes studied respond rapidly (in $1 \mathrm{~min}$ ) in different concentrations of fluoride solutions. The anion-ionophore complex constants in the membrane phase determined using the segmented sandwich membrane method correlate well with the solution-phase binding data and determined selectivity sequence of the ion-selective electrodes. The possibility of real life application of the optimized electrodes was assessed by determination of fluoride concentrations in tap water.

KEYWORDS: polymeric membrane electrode, fluoride, organo-antimony, ionophore, potentiometric

$\mathrm{P}$ olymeric membrane ion-selective electrodes (ISEs) doped with ionophores to prompt the phase transfer of target ions have witnessed exponential growth in recent years owing to their routine utility in environmental, industrial, and clinical fields. $^{1-3}$ Actually, the performance of ISEs is largely dependent on the synthetic ionophores that demonstrate selective binding with target ions and enhanced compatibility with the membrane medium. ${ }^{4-6}$ While the design of cationselective ionophores has experienced significant progress in previous decades, development of highly selective anion ionophores remains a formidable challenge, mainly due to the broader geometrical diversity of anions, larger sizes with relatively low charge densities, and often their inherently strong hydration. ${ }^{7-9}$ Because of their important roles in biological, industrial, and environmental processes, an ability to selectively detect anions is of paramount interest for many applications. Among these anions, fluoride has received much attention considering both their beneficial effects in dental health and adverse effects when excessive intake occurs. The lowering of the recommended fluoride levels from 1.2 to 0.7 ppm by the U.S. Department of Health and Human Services resulted in a renewed interest in developing sensors for aqueous fluoride detection. ${ }^{10}$

Due to its small size, fluoride exhibits highly negative hydration energy, arising from the formation of hydrogen bonds with as many as seven water molecules. ${ }^{11,12}$ Owing to the stability of this solvation shell, fluoride tends to be one of the most difficult ions to be captured in aqueous media. ${ }^{11-13}$ The most popular potentiometric sensor for fluoride determination is a crystalline electrode based on the $\mathrm{LaF}_{3} /$ EuF $_{2}$ membrane. ${ }^{14,15}$ The performance of these electrodes is satisfying; however, it is difficult to miniaturize. Various types

Received: July 19, 2020

Accepted: October 16, 2020

Published: October 28, 2020 
of functional groups or interactions have been utilized in designing anion-selective ionophores, such as Lewis acidic metal atoms and hydrogen bonding. ${ }^{16-18}$ The latter one can be used with anions that possess relatively high charge densities, such as fluoride, which can form hydrogen bonds even with $\mathrm{CH}$ donors polarized with highly withdrawing $\mathrm{CF}_{2}$ groups, leading to the serendipitous discovery of a hydrogen bondingbased macrocyclic fluoride ionophore. ${ }^{16-18}$ By anchoring Lewis acidic metal atoms (e.g., $\mathrm{Sn}(\mathrm{IV}),{ }^{19} \mathrm{Zr}(\mathrm{IV}),{ }^{11,13} \mathrm{Al}-$ (III), ${ }^{20-25} \mathrm{Ge}(\mathrm{IV}),{ }^{15} \mathrm{Cd}(\mathrm{II}),{ }^{26}$ and so on) in the cavity of a macromolecule such as metalloporphyrins and metallophthalocyanines, another kind of most successful fluoride ionophores can be constructed, the selectivity of which is mainly manipulated by the selective binding of fluoride with a central metal. It has been reported that $\mathrm{Zr}(\mathrm{IV})$-based ionophores can operate in membranes via either a charged or/and neutral carrier mechanism, and membranes formulated with $\mathrm{Al}$ (III) octaethylporphyrin display high selectivity. ${ }^{11,20}$ It should be mentioned that, however, problems such as nonNernstian and sluggish response are often observed for the electrodes formulated with some metalloporphyrins ionophores, and development of fluoride-selective ionophores is an ongoing challenge.

Along with the line using Lewis acids as anion ionophores and the recent advances of organo-group 15 Lewis acids in various areas of chemistry, several kinds of Lewis acidic organo-antimony $(\mathrm{V})$ compounds have been reported to bind fluoride with high affinity and selectivity in the solution phase, so it is very interesting to see whether these compounds could be used as potential fluoride-selective ionophores to fabricate corresponding electrodes. ${ }^{27-32}$ Here, we introduced four Lewis acidic organo-antimony $(\mathrm{V})$ compounds as new ionophores incorporated into fluoride-selective polymeric membrane electrodes. The performance of the ionophores was fully evaluated; after optimization of the membrane compositions such as the concentrations of ionophores, plasticizers, and lipophilic additives, enhanced detection limits and excellent selectivity can be obtained. The selectivity coefficients were determined and compared to the binding constants calculated from the segmented sandwich membrane method for the corresponding ionophore-fluoride complexes in the membrane. Moreover, ISEs with optimal performance offer a simple alternative for fluoride sensing in real world situations.

\section{EXPERIMENTAL SECTION}

Reagents. The organo-antimony $(\mathrm{V})$ compounds 1-4 were supplied by Qingdao Zhongke Chemicals (prepared as described in the literature). ${ }^{10,12,33,34}$ It should be noticed that compound $\mathbf{1}$ and $\mathbf{2}$ were obtained as its $\left[\mathrm{Ph}_{4} \mathrm{Sb}\right] \mathrm{Br}$ and $\left[\mathrm{Ph}_{4} \mathrm{Sb}\right] \mathrm{OTf}$ slats, respectively. High molecular weight poly(vinyl chloride) (PVC), 2-nitrophenyl octyl ether (o-NPOE), bis(2-ethylhexyl) sebacate (DOS), potassium tetrakis[3,5-bis(trifluoro-methyl)phenyl]borate (KTFPB), and tridodecylmethylammonium chloride (TDMACl) were purchased from Sigma. Glycine (Gly) and 4-morpholinoethanesulfonic acid (MES) were purchased from Fluka. Other reagents were purchased from Sinopharm Group Co., Ltd. All chemicals were of selectophore or analytical reagent grade. Aqueous solutions were prepared with freshly deionized water (18.2 $\mathrm{M} \Omega \mathrm{cm}$ specific resistance) obtained with a Pall Cascada laboratory water system.

Membranes and Electrode Preparation. Polymeric membrane electrodes were prepared by using a solvent-casting technique according to the established procedures reported elsewhere. ${ }^{6}$ Briefly, 1.0 wt \% organo-antimony $(\mathrm{V})$ compounds $1-4$, TDMACl or KTFPB (mol \% relative to the ionophore), and either NPOE or DOS plasticizers (66.0 wt \%) as well as PVC (33.0 wt \%) were dissolved in
THF to prepare membrane cocktails. After transferring the membrane cocktail to a glass ring fixed on a glass plate and evaporation of the tetrahydrofuran overnight, a uniform polymeric liquid membrane (ca. $200 \mu \mathrm{m}$ in thickness) was obtained. Subsequently, the as-formed membrane was cut into small disks of $7 \mathrm{~mm}$ diameter using a cork borer to install onto Philips IS-561 electrode bodies. A solution of Gly $/ \mathrm{H}_{3} \mathrm{PO}_{4}(200 \mathrm{mM}, \mathrm{pH}=3.0)$ buffer with the addition of $\mathrm{NaF}(1$ $\mathrm{mM}$ ) was used as the internal filling solution. It should be noticed that a majority of fluoride is present in its protonated form at $\mathrm{pH} 3$. Due to its special skin penetrating and bone dissolving properties, special attention is necessary. Membranes (both of the two sides) were conditioned in the internal filling solution overnight before evaluating the potentiometric performance.

To prepare the sandwich membranes, two plasticized PVC membranes with or without ionophores (the first one is based on ion exchangers (1.0 wt \%) only, and the second one contains the given ionophore (1.0 wt \%) and TDMACl or KTFPB (50.0 mol \% relative to the ionophore)) were conditioned separately for two days in $1 \times 10^{-2} \mathrm{M}$ solution of specific anions prepared with $\mathrm{Gly} / \mathrm{H}_{3} \mathrm{PO}_{4}$ $(200 \mathrm{mM}, \mathrm{pH}=3.0)$ buffer. After drying with filter paper, the sandwich membrane was then assembled by attaching the membrane with an ionophore to the ionophore-free membrane. The sandwich membrane was then mounted immediately in the electrode body.

Potentiometric Measurements. Potentiometric measurements were carried out using the $\mathrm{CHI} 660 \mathrm{C}$ electrochemical station (Shanghai Chenhua Apparatus) at $22.0{ }^{\circ} \mathrm{C}$ with $\mathrm{Ag} / \mathrm{AgCl}(3 \mathrm{M}$ $\mathrm{KCl})$ as the reference electrode in the galvanic cell: $\mathrm{Ag} / \mathrm{AgCl} 3.0 \mathrm{M}$ $\mathrm{KClll} 1.0 \mathrm{M} \mathrm{CH}_{3} \mathrm{COOLillsample} \mathrm{solution|ISE} \mathrm{membranelinner} \mathrm{filling}$ solution $/ \mathrm{Ag} / \mathrm{AgCl}$. The selectivity coefficients were detected by the separate solution method (SSM), and complex formation constants were determined by the method described by Bakker. ${ }^{35}$ The selectivity coefficients were calculated using the potential values at high concentrations of anions assuming theoretical slopes. The proposed electrode was used for determination of fluoride concentrations in tap water adjusted to $\mathrm{pH} 3.0$ using $200 \mathrm{mM}$ Gly/ $\mathrm{H}_{3} \mathrm{PO}_{4}$ buffer. All the experimental values were obtained with three replications.

\section{RESULTS AND DISCUSSION}

Along with the line using Lewis acids as anion ionophores and the recent advances of organo-group 15 Lewis acids in supramolecular chemistry, we questioned whether fluorideselective polymeric membrane electrodes using pnictonium ions $\left(\left[\mathrm{R}_{4} \mathrm{Pn}\right]^{+}, \mathrm{Pn}=\right.$ pnictogen $)$ as ionophores could be envisaged. ${ }^{27-29}$ Actually, pnictonium ions such as $\left[\mathrm{R}_{4} \mathrm{P}\right]^{+}$bind fluoride in strictly anhydrous and nonpolar media, and it has been reported that $\left[\mathrm{Ph}_{4} \mathrm{Sb}\right]^{+}(\mathbf{1}$ in Scheme 1) displayed high fluoride affinity in biphasic $\mathrm{CCl}_{4} / \mathrm{H}_{2} \mathrm{O}$ mixtures. ${ }^{30-32}$ Further investigation of the fluoride binding process with 1 in acetonitrile showed a binding constant greater than $10^{6} \mathrm{M}^{-1}$ and a change of the coordination geometry of antimony from tetrahedral in 1 to trigonal-bipyramidal in $\mathbf{1}-\mathrm{F}^{-} .{ }^{10,27}$ Encouraged by previous results, a highly acidic and air stable $\left[\mathrm{Sb}\left(\mathrm{C}_{6} \mathrm{~F}_{5}\right)_{4}\right]^{+}$(2 in Scheme 1) has also been synthesized by anchoring strong electron withdrawing groups on the ligands. Although no exact fluoride binding constant was reported for 2, elimination of the fluoride ligand from $\mathrm{SbF}_{5}$ and $\mathrm{B}\left(\mathrm{C}_{6} \mathrm{~F}_{5}\right)_{3}$ (with fluoride affinities of 489 and $444 \mathrm{~kJ} / \mathrm{mol}$, respectively) was observed, demonstrating its high fluoride affinity. ${ }^{30}$ For the stibonium ions mentioned before, it is obvious that Coulombic effects which drive the ion pairing process contribute significantly to the high fluoride binding affinity, and ion pairing may deteriorate the selectivity of the ionophores especially for high charge interfering ions, so it is very interesting to see whether neutral organoantimony $(\mathrm{V})$ compounds would be sufficiently Lewis acidic to complex 
Scheme 1. Structures of Organo-Antimony(V) Compounds Used as Fluoride Ionophores in This Work

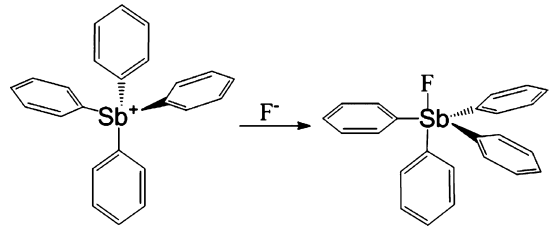

1

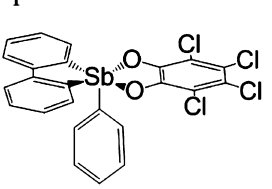

3

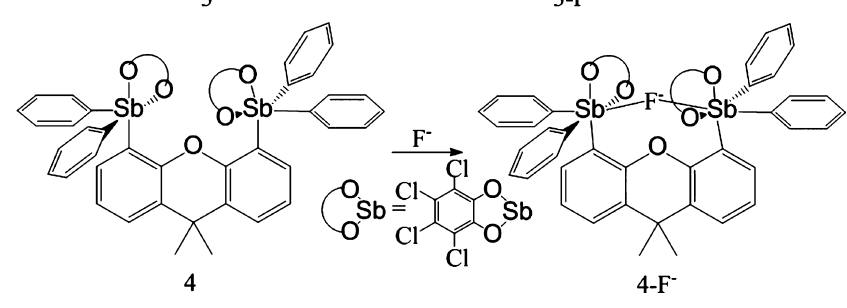

fluoride with high affinity in the polymeric membrane phase. Considering that spirocyclic stiboranes exhibit great structural stability and less hindered access to the antimony atom, the fluoride binding affinity of tetrachloro-substituted organoantimony $(\mathrm{V})$ compounds (3 in Scheme 1) has been inspected; titration experiment in $\mathrm{THF} / \mathrm{H}_{2} \mathrm{O}(7: 3, \mathrm{v} / \mathrm{v})$ solution exhibited a stability constant of $13500( \pm 1400)$ $\mathrm{M}^{-1}$ for $3-\mathrm{F}^{-31}$ Moreover, with the experience of diboranebased anion receptors, it is reasonable to deploy two stiborane units in a face-to-face fashion, and thus, a bidentate ionophore with a cavity flanked on either side by Lewis acidic antimony(V) atoms (4 in Scheme 1) can be developed. Very impressive binding affinity for fluoride with this new bidentate receptor in aqueous solutions $\left(95 \% \mathrm{H}_{2} \mathrm{O}, 700 \pm 30 \mathrm{M}^{-1}\right)$ was observed. ${ }^{12}$ Considering the relatively high binding constant of Lewis acidic organo-antimony(V) compounds 1-4 with fluoride in the solution phase, we hypothesized that they could be good candidates as charged or neutral ionophores for fluoride. To assess this possibility, membranes formulated with different Lewis acidic organo-antimony(V) compounds, lipophilic additives, and plasticizers were prepared, and their potentiometric responses to fluoride and potential interfering anions were evaluated for optimal performance (Table 1). $\mathrm{Gly} / \mathrm{H}_{3} \mathrm{PO}_{4}$ buffer ( $\mathrm{pH}$ 3.0) was used as the background electrolyte solution since fluoride-selective electrodes reported previously display good performance in this solution.

As shown in Table 1 and Figure 1, all four ionophores could yield membrane electrodes that exhibit Nernstian/nearNernstian response to fluoride and potentiometric selectivity patterns different significantly from Hofmeister series, confirming the strong ionophore properties and high affinity of fluoride to compounds 1-4. Knowledge of the working mechanism of ionophores is a very important factor when optimizing membrane composition. ${ }^{21}$ It has been reported previously that metalloporphyrins incorporated into fluorideselective membranes could function as both charged and neutral carriers and, thus, lipophilic anionic or cationic additives are required, respectively. ${ }^{11,13,35}$ Experiments without additives and with anionic and cationic additives for all studied ionophores have been performed to give the exact description of the response mechanism (Table 1). Considering that antimony is in a +5 oxidation state and could accommodate as many as six ligands, a charge and neutral carrier mechanism could both be envisioned in ionophores 1 and 2, depending on whether the first axial ligand $\left(\mathrm{Br}^{-}\right.$and $\mathrm{OTf}^{-}$for ionophores 1 and 2, respectively) exhibits strong enough ligation to the antimony (V) center. To determine which kind of mechanism operates in the case of the charged ionophores 1 reported herein, ISEs containing $30 \mathrm{~mol} \%$ of anionic (ISE III) or cationic additives (ISE VI) were prepared. The observed antiHofmeister selectivity sequence of $\mathrm{F}^{-}>\mathrm{ClO}_{4}{ }^{-} \approx \mathrm{SCN}^{-}>\mathrm{I}^{-}>$ $\mathrm{NO}_{2}{ }^{-} \approx \mathrm{NO}_{3}{ }^{-}>\mathrm{Cl}^{-}>\mathrm{Br}^{-}$for ISE III differs significantly from that for ISE VI (Hofmeister series) possessing cationic sites, demonstrating that the ionophores 1 serve as the charged ionophore only. Although a little counterintuitive (considering that the maximum coordination number is 6 , it seems that ionophore 1 could also function as a neutral ionophore), these results correlate well with the observation that no further spectral change was found after an equivalent of fluoride was added to acetonitrile solution of $\mathbf{1}$. $^{10}$ Moreover, selectivity coefficients for membrane electrodes prepared with only ionophores (see ISEs I, VII, X, and XIII), ionophore 2, and TDMACl (see ISE IX), as well as ionophore 4 and KTFPB have also been determined. Obviously, membrane electrodes formulated with only ionophores 1-2 (ISEs I and VII) behave similar in the selectivity pattern with their anionic additives containing the counterpart (ISEs II-IV and VIII), while the membrane fabricated with the same ionophores and cationic additives displayed deteriorated selectivity especially for lipophilic anions such as perchlorate and thiocyanate (ISEs VI and IX). On the other hand, electrodes doped with or without anionic additives and ionophores 3-4 only showed weak anionic responses to fluoride and lipophilic anions, consistent with the large binding affinity of the ionophores (ISEs X, XII, XIII, and XVII). Generally, ion exchangers are added to initiate permselectivity toward anions; however, for strong binding ionophores, a weak anionic response can be achieved even in the absence of ion exchangers. ${ }^{6}$ All these data describe a clear picture of the response mechanism of all the ionophores, ionophores $\mathbf{1 - 2}$ are governed by charged carrier mechanisms, while ionophores 3-4 function as neutral anion carriers. Actually, $\left[\mathrm{Ph}_{4} \mathrm{Sb}\right] \mathrm{Br}$ may adopt a partial ionic structure in the membrane phase. ${ }^{33}$

The influence of the lipophilic additive content on the performance of the electrodes prepared with the $\mathrm{PVC} / \mathrm{o}$ NPOE membranes containing different ionophores (using ionophores $\mathbf{1}$ and $\mathbf{4}$ as examples, ISEs II-IV and XIV-XVI) was assessed. While little differences in the slopes of ISEs IIIV when changing the amount of additives are observed, ISE II containing $10 \mathrm{~mol} \%$ of KTFPB showed the best detection limits. On the other hand, the amount of TDMACl does have a significant influence on the slopes of natural carrier-based ISEs XIV-XVI; $50 \mathrm{~mol} \%$ is necessary to obtain Nernstian response. Moreover, for membranes formulated with compound ionophores 1 and 4, optimal selectivity was observed when $30 \mathrm{~mol} \% \mathrm{KTFPB}$ and $50 \mathrm{~mol} \%$ TDMACl were used as lipophilic additives, respectively. Unfortunately, electrodes with optimized fluoride selectivity (ISE III and ISE XVI) did not have the best detection limits (ISE II and ISE XIV). Actually, lipophilic additives could increase the polarity of the membrane, which enhances the phase transfer of hydrophilic anions. ${ }^{5}$ However, the detection limit might be elevated owing 
要

|ż⿺辶一兀

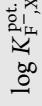

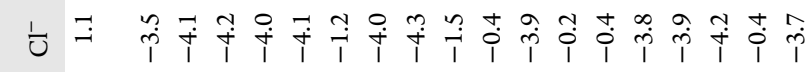

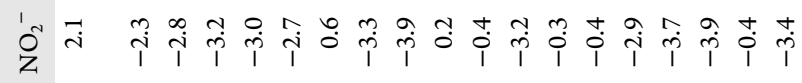

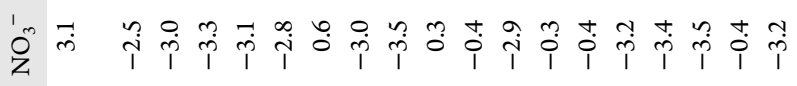

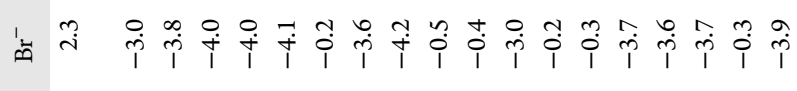

总

方的

L

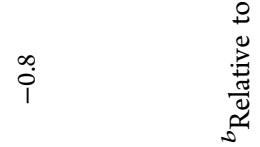

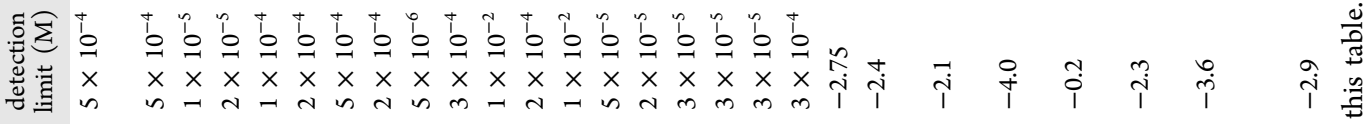

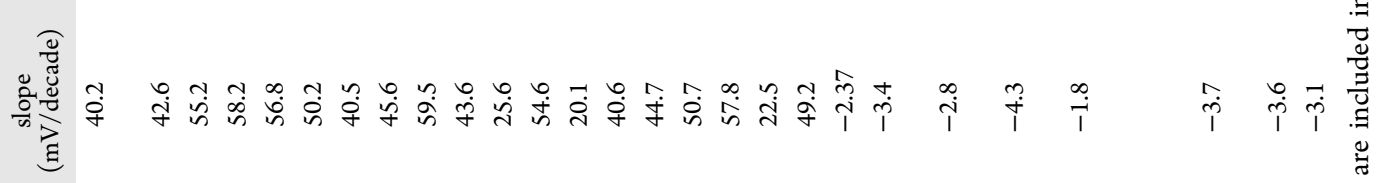

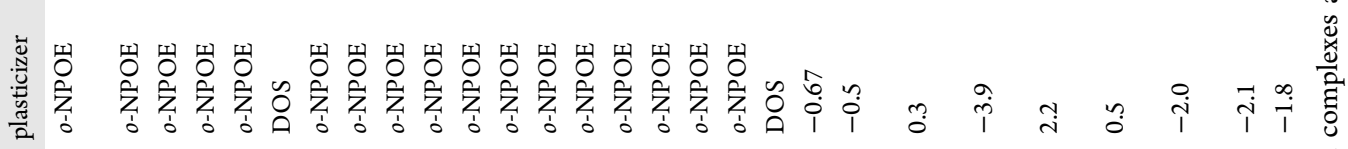
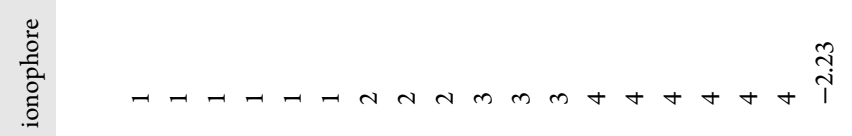

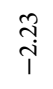

\section{吾}

僁
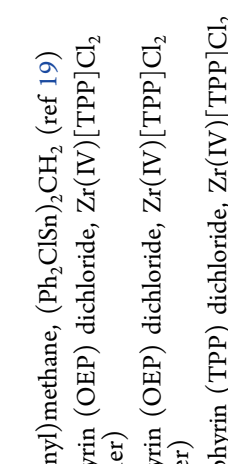

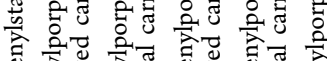



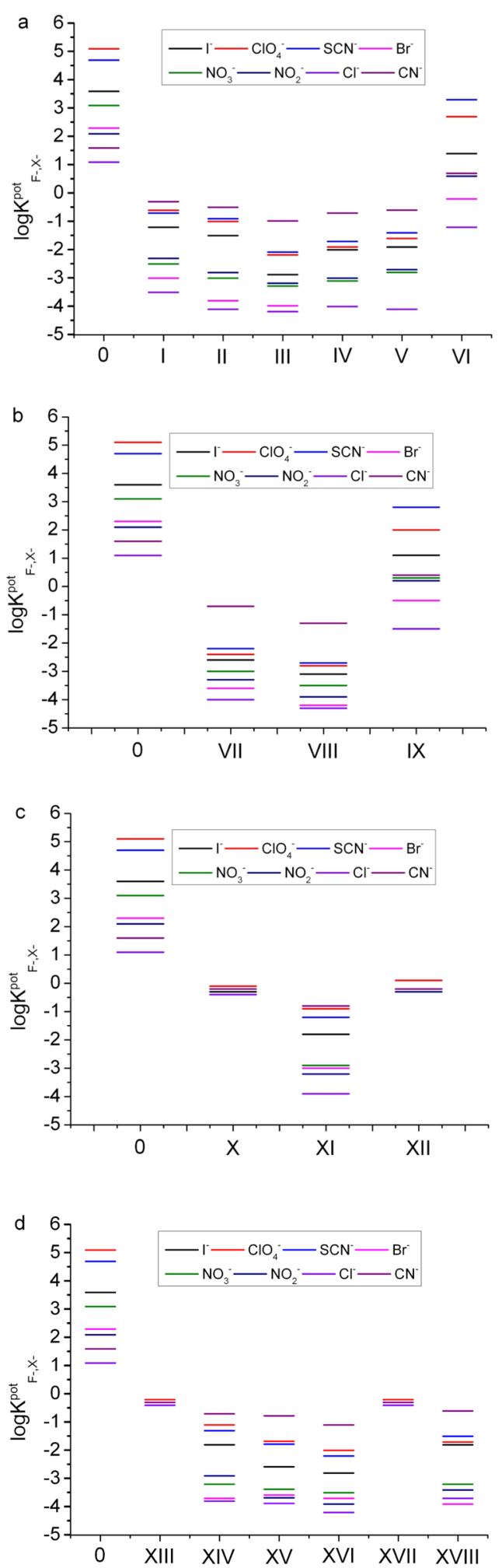

Figure 1. Potentiometric selectivity pattern of the membrane electrodes fabricated with ionophores 1-4 $(a-d)$ toward fluoride over different anions.

to the increase of the ion flux across the membrane with increasing lipophilic additives. ${ }^{16}$ For all the ISEs tested (except for ISEs VI, X, XII, XIII, and XVII), a similar anti-Hofmeister selectivity sequence is obtained; the selectivity coefficients for fluoride are very high, and the discrimination over other interfering anions is relatively strong. It is obvious that ISE VIII displayed the best selectivity and detection limit, which is comparable or superior to all of the previously reported polymeric membrane fluoride-selective electrodes (Table 1). ${ }^{11,13,15,19,21,26,36}$ Actually, only $\mathrm{Zr}(\mathrm{IV})$-tetraphenylporphyrin (TPP) dichloride operated under the mechanism of the charged carrier has been reported to show superior selectivity than the values reported here. However, deteriorated detection limit $\left(10^{-4.5} \mathrm{M}\right)$, super-Nernstian response, and short life-time (one month or even 3-4 days claimed by the authors) prohibited it from practical application. ${ }^{11}$ It should be noticed that the proposed ISEs also showed good selectivity to fluoride over cyanide (determined in MES/ $\mathrm{NaOH}$ buffer solution ( $\mathrm{pH}$ 5.5) owing to the low acidity of cyanic acid), which has been reported to be more lipophilic and complex organo-antimony(V) compounds with high affinity. ${ }^{37,38}$ The influence of a plasticizer on the performance of the ISEs should also be noticed; membranes plasticized with more polar $o$-NPOE showed a better slope and selectivity toward fluoride over interfering anions compared to membranes containing DOS. This result can be ascribed to the difference in the dielectric constant of the plasticizers. A higher dielectric constant decreases the electrical resistance of the membrane and facilitates the transfer of hydrophilic ions to the membrane phase. ${ }^{16}$ It should be noted that these selectivity coefficients were determined by the SSM assuming theoretical slopes; an analogous approach has been used previously, which makes it possible to compare the selectivity coefficient data of the new ionophores with that of other ionophores reported earlier. In parallel, the potentiometric calibration curves toward various anions are also illustrated in Figure 2.

Due to the high affinity of $\mathrm{OH}^{-}$anions to metal ions, the $\mathrm{pH}$ of the sample solutions may have significant influence on the performance of ISEs using Lewis acidic organo-antimony(V) compounds as ionophores in this work. Thus, the effects of sample solution $\mathrm{pH}$ on the fluoride response characteristics of ISE III, VIII, XI, and XVI were inspected in detail. For this purpose, in addition to a Gly $/ \mathrm{H}_{3} \mathrm{PO}_{4}$ buffer ( $\mathrm{pH} 3.0$ ), a MES/ $\mathrm{NaOH}$ buffer solution ( $\mathrm{pH} 5.5)$ was also examined as the background electrolyte solutions for potentiometric fluoride determination (See Figure 3). For all the four ISEs examined, increasing sample $\mathrm{pH}$ from 3.0 to 5.5 deteriorates the detection limits, slopes, and linear response ranges of the ISEs. For example, for ISE VIII plasticized with o-NPOE using compound 2 as the ionophore along with $30 \mathrm{~mol} \%$ of KTFPB, the detection limits shift significantly from $5 \times 10^{-6}$ to $4 \times 10^{-5} \mathrm{M}$. A similar behavior is observed for ISEs III, XI, and XVI. Generally, it was found that a change of $\mathrm{pH}$ from 3.0 to 5.5 increases the LDL by at least one order of magnitude. To give more information about the influence of $\mathrm{OH}^{-}$, potential data in $10^{-2} \mathrm{M}$ fluoride solution ( $\mathrm{pH} 3.0$ ) and that in HEPES buffer $(\mathrm{pH}$ 7.0) were used to calculate the selectivity coefficient over $\mathrm{OH}^{-}$, and the selectivity values for ISEs III, VIII, XI, and XVI are 5.0, 4.8, 4.2, and 4.1, respectively. Although an improved detection limit for the proposed ISEs can be anticipated at $\mathrm{pH} 3.0$, these values correlate with the detection limit at $\mathrm{pH}$ 5.5. Plainly, the Gly/ $\mathrm{H}_{3} \mathrm{PO}_{4}$ buffer $(\mathrm{pH}$ 3.0) is preferred for practical application of these new fluoride ISEs.

Examples of the dynamic responses of representative ISE VIII and XVI to fluoride are presented in Figure 4. In contrast to the sluggish response for previously reported polymeric membrane fluoride-selective electrodes, dramatically enhanced dynamic response for the ISEs developed in this work was 

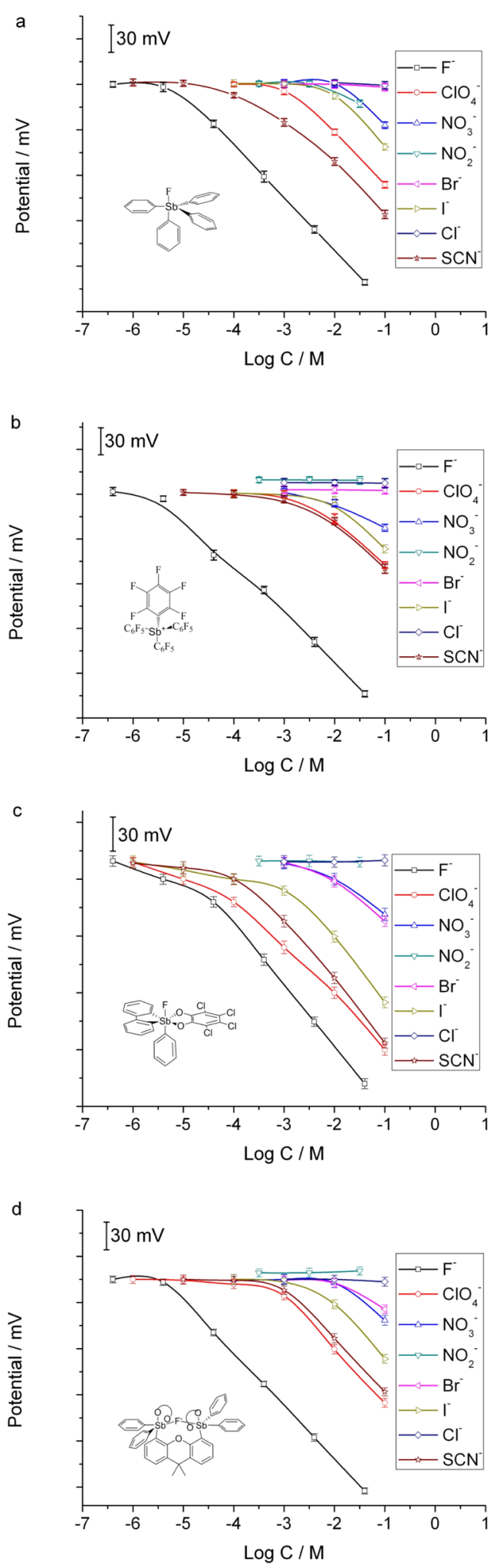

Figure 2. Potentiometric response of ISE III (a), VIII (b), XI (c), and $\mathrm{XVI}(\mathrm{d})$ toward different anions.

observed. Although a little potential drift (ca. $5.0 \mathrm{mV}$ ) was observed for dilute fluoride solutions, response times were considerably shorter $(<60 \mathrm{~s})$. Reversibility of the ISEs is very important especially when the binding affinity is high. As required, potential traces when switching sensors from high to low concentrations for several times are displayed in Figure 4.

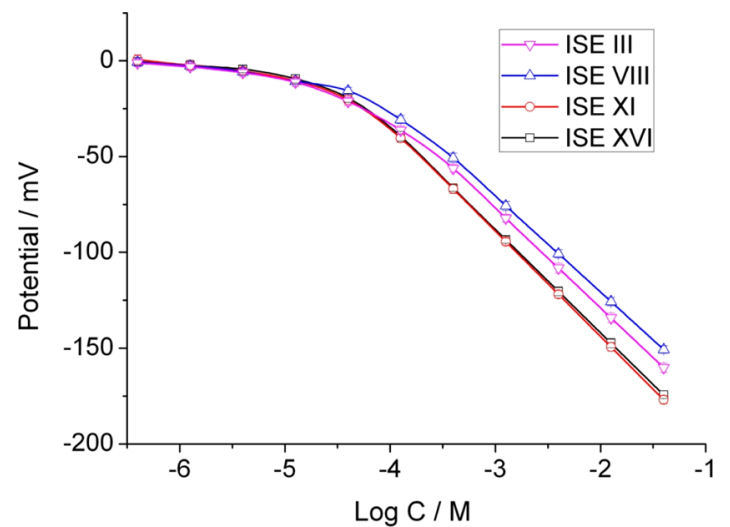

Figure 3. Potentiometric response of ISEs III, VIII, XI, and XVI toward fluoride in MES/ $\mathrm{NaOH}$ buffer solution ( $\mathrm{pH} 5.5$ ).

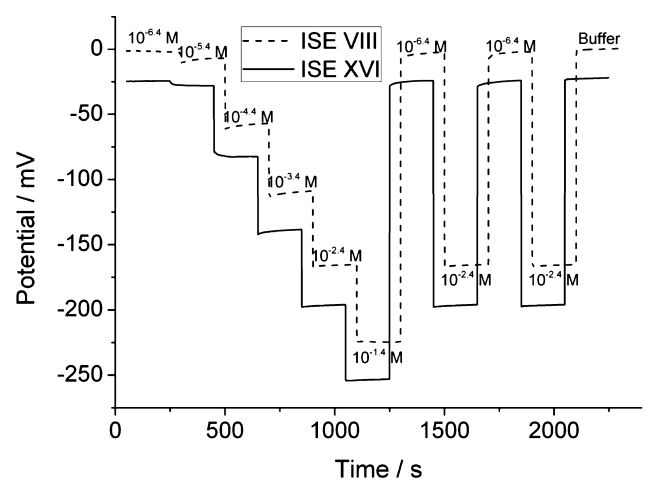

Figure 4. Dynamic fluoride EMF response of ISE VIII and XVI. Measurements were carried out in $\mathrm{Gly} / \mathrm{H}_{3} \mathrm{PO}_{4}$ buffer $(\mathrm{pH}=3.0)$.

It seems that good reversibility is obtained, although strong complexation with fluoride is observed; similar phenomena have also been reported in previous work. ${ }^{21}$ The stability of the developed electrodes has been tested over a period of 3 months; no significant changes in the slopes, detection limits, and selectivity coefficients were observed (see Figure S1 in Supporting Information). Moreover, $\log P$ values for ionophores 1-4 have been calculated using ChemDraw 2014; the obtained values are 7.17, 9.19, 9.51, and 19.90 for ionophores $1-4$, respectively, correlating well with the stability of the corresponding membrane electrodes.

It is well-known that the binding constants of ion-ionophore complexes are critical for the selectivity of polymeric membrane ISEs. Although the solution-phase binding data for fluoride and ionophores $\mathbf{1 - 4}$ is available (mentioned previously in the introduction), it is difficult to translate these data directly to the performance of the ionophore-based ISEs because the membrane environment differs significantly from a simple solvent. Fortunately, the segmented sandwich method proposed by Bakker enables the determination of ionionophore stability constants within the membrane phase. The values of the formation constants for various anions with the four different ionophores in $o$-NPOE-plasticized membranes are shown in Table 2. It is not surprising that each of the ionophores form their strongest complexes with fluoride and the trend in the stability constants of the fluorideionophore complex correlates well with the solution-phase binding data and the determined selectivity sequence of the ISEs. It should also be noticed that the significant difference between the formation constants for fluoride and other 
Table 2. Ionophore Complex Formation Constants Determined with Two-Segment PVC/o-NPOE (1:2) Sandwich Membranes $^{a}$

\begin{tabular}{|c|c|c|c|c|c|c|c|c|c|}
\hline \multirow[b]{2}{*}{ ionophore (1 wt \%) } & \multirow[b]{2}{*}{ ionic sites (mol \%) } & \multicolumn{8}{|c|}{ formation constants $\left(\log K_{\mathrm{a}}\right)$} \\
\hline & & $\mathrm{F}^{-}$ & $\mathrm{I}^{-}$ & $\mathrm{ClO}_{4}^{-}$ & $\mathrm{SCN}^{-}$ & $\mathrm{Br}^{-}$ & $\mathrm{NO}_{3}^{-}$ & $\mathrm{NO}_{2}^{-}$ & $\mathrm{Cl}^{-}$ \\
\hline 1 & KTFPB & 11.1 & 4.7 & 4.1 & 4.2 & 4.7 & 4.4 & 4.6 & 5.0 \\
\hline 2 & KTFPB & 12.2 & 5.0 & 4.2 & 4.4 & 5.1 & 4.8 & 4.9 & 5.3 \\
\hline 3 & TDMACl & 9.9 & 4.7 & 4.0 & 4.0 & 4.8 & 4.3 & 4.3 & 4.9 \\
\hline 4 & TDMACl & 10.8 & 4.3 & 3.9 & 4.0 & 4.5 & 4.1 & 4.0 & 5.0 \\
\hline
\end{tabular}

${ }^{a_{\text {The }}}$ complex stoichiometry is $1: 1$.

interring ions does not translate equivalently to the selectivity. Using ionophore $\mathbf{2}$ as an example, we observe that the difference between the formation constant values for fluoride and perchlorate is 8.0, whereas $\log K_{\mathrm{F}^{-}}^{\text {pot. }} \mathrm{ClO}_{4}^{-}$is -2.8 . This difference could be ascribed to coextraction, different thermodynamics of ions, and ion-exchange processes that generate an ion flux across the membrane. ${ }^{16}$ Actually, the ratio of the complex formation constants of an ionophore with various ions should directly manifest itself as a change in selectivity, as compared to Hofmeister series. It is well-known that electron withdrawing groups could increase the Lewis acidity of the metal ion, so it is reasonable that ionophore 2 with perfluorinated substituents complex stronger with all anions than ionophore 1 . When comparing the anion complexation constants of ionophores $3-4$ to $1-2$, it is clear that stronger complexes are formed by the charged organoantimony (V) derivatives.

As described above, the performance (with a linear range of $1 \times 10^{-5}$ to $4 \times 10^{-2} \mathrm{M}$ and a detection limit of $5 \times 10^{-6} \mathrm{M}$ ) of ISE VIII suggests that it may suit for real-life applications. Therefore, fluoride detection in tap water was performed using ISE VIII and commercial $\mathrm{LaF}_{3}$ electrodes. As shown in Table 3, data obtained using ISE VIII are consistent with those obtained using the standard $\mathrm{LaF}_{3}$ electrode.

Table 3. Analytical Results for the Detection of Fluoride in Tap Water Samples (Mean \pm Standard Deviation, $n=3$ )

\begin{tabular}{ccc} 
samples & ISE VIII $\left(10^{-5} \mathrm{M}\right)$ & $\mathrm{LaF}_{3}$ ISE $\left(10^{-5} \mathrm{M}\right)$ \\
tap water 1 & $3.25 \pm 0.10$ & $3.24 \pm 0.07$ \\
tap water 2 & $2.73 \pm 0.09$ & $2.74 \pm 0.07$ \\
tap water 3 & $2.55 \pm 0.09$ & $2.54 \pm 0.08$ \\
tap water 4 & $1.95 \pm 0.08$ & $2.03 \pm 0.06$ \\
tap water 5 & $2.23 \pm 0.07$ & $2.18 \pm 0.07$ \\
\hline
\end{tabular}

\section{CONCLUSIONS}

Four kinds of Lewis acidic organo-antimony(V) compounds with strong binding affinity to fluoride were used as ionophores to develop polymeric membrane fluoride-selective electrodes that show significant anti-Hofmeister selectivity. These new ionophores function via the charged (ionophores 1 and 2) or neutral (ionophores 3 and 4) carrier mechanism. Improved detection limits and enhanced selectivity could be achieved by optimizing the ionophores, lipophilic additives, and plasticizers. Membrane electrodes fabricated with tetrakis(pentafluorophenyl)stibonium (ionophore 2) performed best in detection limit, sensitivity, and selectivity. The optimized electrodes demonstrated a response toward fluoride with a slope of $-59.5 \mathrm{mV} /$ decade in the linear range of $1 \times 10^{-5}$ to 4 $\times 10^{-2} \mathrm{M}$ and a detection limit of $5 \times 10^{-6} \mathrm{M}$. Studies on the influence of sample solution $\mathrm{pH}$ indicate that the best $\mathrm{pH}$ for fluoride determination is $\mathrm{pH}$ 3.0. Moreover, all of the electrodes studied respond in $1 \mathrm{~min}$. The anion-ionophore complex constants in the membrane phase determined using the segmented sandwich membrane method correlate well with the solution-phase binding data and the determined selectivity sequence of the ISEs. The optimized electrodes can be used for the determination of fluoride concentrations in tap water.

\section{ASSOCIATED CONTENT}

SI Supporting Information

The Supporting Information is available free of charge at https://pubs.acs.org/doi/10.1021/acssensors.0c01481.

Data for slopes, detection limits, and selectivity coefficients in a period of 3 months (PDF)

\section{AUTHOR INFORMATION}

\section{Corresponding Author}

Long Li - College of Environment and Safety Engineering, Qingdao University of Science and Technology, Qingdao

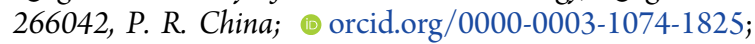
Phone: +86 532 84022020; Email: lilongyln@yeah.net

\section{Authors}

Yihao Zhang - College of Environment and Safety Engineering, Qingdao University of Science and Technology, Qingdao 266042, P. R. China

Ying Li - College of Environment and Safety Engineering, Qingdao University of Science and Technology, Qingdao 266042, P. R. China

Yinghui Duan - College of Chemical Engineering, Qingdao University of Science and Technology, Qingdao 266042, P. R. China

Yi Qian - College of Chemical Engineering, Qingdao University of Science and Technology, Qingdao 266042, P. R. China

Peidong Zhang - College of Environment and Safety Engineering, Qingdao University of Science and Technology, Qingdao 266042, P. R. China

Qingjie Guo - State Key Laboratory of High-Efficiency Utilization of Coal and Green Chemical Engineering, Ningxia University, Yinchuan 750021, P. R. China

Jiawang Ding - Key Laboratory of Coastal Environmental Processes and Ecological Remediation and Shandong Provincial Key Laboratory of Coastal Environmental Processes, Yantai Institute of Coastal Zone Research (YIC), Chinese Academy of Sciences (CAS), Yantai 264003, Shandong, P. R. China; Laboratory for Marine Biology and Biotechnology, Qingdao National Laboratory for Marine Science and Technology, Qingdao 266200, P. R. China

Complete contact information is available at: https://pubs.acs.org/10.1021/acssensors.0c01481 


\section{Notes}

The authors declare no competing financial interest.

\section{ACKNOWLEDGMENTS}

This work was financially supported by the Key R \& D Project of Shandong Province (no. 2019CSF109001 and 2019CSF109080), the Shandong Provincial Natural Science Foundation, China (no. ZR2018BB072), the Original Innovation Project of Qingdao City (no. 19-6-2-23-cg), the Foundation of State Key Laboratory of High-efficiency Utilization of Coal and Green Chemical Engineering (no. 2018-K09 and 2018-K43), the Key Laboratory of Coastal Environmental Processes and Ecological Remediation, YICCAS (no. 2018KFJJ02), the Opening Project of Shandong Ecochemical Engineering Collaborative Innovation Center (no. XTCXQN02), and the Open Project of Chemistry Department of Qingdao University of Science and Technology (no. QUSTHX201920).

\section{REFERENCES}

(1) Zdrachek, E.; Bakker, E. Potentiometric sensing. Anal. Chem. 2019, 91, 2-26.

(2) Bakker, E. Electroanalysis with membrane electrodes and liquidliquid interfaces. Anal. Chem. 2016, 88, 395-413.

(3) Ding, J.; Qin, W. Recent advances in potentiometric biosensors. Trac-Trend. Anal. Chem. 2020, 124, 115803.

(4) Hein, R.; Beer, P. D.; Davis, J. J. Electrochemical anion sensing: supramolecular approaches. Chem. Rev. 2020, 120, 1888-1935.

(5) Zahran, E. M.; Hua, Y.; Li, Y.; Flood, A. H.; Bachas, L. G. Triazolophanes: a new class of halide-selective ionophores for potentiometric sensors. Anal. Chem. 2010, 82, 368-375.

(6) Zahran, E. M.; Fatila, E. M.; Chen, C.-H.; Flood, A. H.; Bachas, L. G. Cyanostar: C-H hydrogen bonding neutral carrier scaffold for anion-selective sensors. Anal. Chem. 2018, 90, 1925-1933.

(7) Langton, M. J.; Serpell, C. J.; Beer, P. D. Anion recognition in water: recent advances from a supramolecular and macromolecular perspective. Angew. Chem., Int. Ed. 2016, 55, 1974-1987.

(8) Sabek, J.; Adriaenssens, L.; Guinovart, T.; Parra, E. J.; Rius, F. X.; Ballester, P.; Blondeau, P. Chloride-selective electrodes based on "two-wall" aryl-extended calix[4]pyrroles: combining hydrogen bonds and anion-pi interactions to achieve optimum performance. Chem.Eur. J. 2015, 21, 448-454.

(9) Cuartero, M.; Ortuño, J. A.; García, M. S.; Sánchez, G.; MásMontoya, M.; Curiel, D. Benzodipyrrole derivates as new ionophores for anion-selective electrodes: Improving potentiometric selectivity towards divalent anions. Talanta 2011, 85, 1876-1881.

(10) Ke, I.-S.; Myahkostupov, M.; Castellano, F. N.; Gabbaï, F. P. Stibonium ions for the fluorescence turn-on sensing of $\mathrm{F}^{-}$in drinking water at parts per million concentrations. J. Am. Chem. Soc. 2012, 134, 15309-15311.

(11) Malinowska, E.; Górski, Ł.; Meyerhoff, M. E. Zirconium(IV)porphyrins as novel ionophores for fluoride-selective polymeric membrane electrodes. Anal. Chim. Acta 2002, 468, 133-141.

(12) Hirai, M.; Gabbaï, F. P. Squeezing fluoride out of water with a neutral bidentate antimony(V) Lewis acid. Angew. Chem., Int. Ed. 2015, 54, 1205-1209.

(13) Górski, L.; Meyerhoff, M. E.; Malinowska, E. Polymeric membrane electrodes with enhanced fluoride selectivity using $\mathrm{Zr}(\mathrm{IV})$ porphyrins functioning as neutral carriers. Talanta 2004, 63, 101107.

(14) Frant, M. S.; Ross, J. W. Electrode for sensing fluoride ion activity in solution. Science 1966, 154, 1553-1555.

(15) Siwiec, K.; Górski, Ł. The application of germanium(IV)porphyrins as fluoride-selective ionophores for polymeric membrane electrodes. J. Electroanal. Chem. 2019, 833, 498-504.

(16) Zahran, E. M.; Hua, Y.; Lee, S.; Flood, A. H.; Bachas, L. G. Ionselective electrodes based on a pyridyl-containing triazolophane: altering halide selectivity by combining dipole-promoted cooperativity with hydrogen bonding. Anal. Chem. 2011, 83, 3455-3461.

(17) Farnham, W. B.; Roe, D. C.; Dixon, D. A.; Calabrese, J. C.; Harlow, R. L. Fluorinated macrocyclic ethers as fluoride ion hosts. Novel structures and dynamic properties. J. Am. Chem. Soc. 1990, 112, $7707-7718$.

(18) Siswanta, D.; Takenaka, J.; Suzuki, T.; Sasakura, H.; Hisamoto, H.; Suzuki, K. Novel neutral anion ionophores based on fluorinated (poly)ether compounds as a sensory molecule for an ion-selective electrode. Chem. Lett. 1997, 26, 195-196.

(19) Perdikaki, K.; Tsagkatakis, I.; Chaniotakis, N. A.; Altmann, R.; Jurkschat, K.; Reeske, G. Selective fluoride recognition and potentiometric properties of ion-selective electrodes based on bis(halodiphenylstannyl)alkanes. Anal. Chim. Acta 2002, 467, 197204.

(20) Badr, I. H. A.; Meyerhoff, M. E. Fluoride-selective optical sensor based on aluminum(III)-octaethylporphyrin in thin polymeric film: further characterization and practical application. Anal. Chem. 2005, 77, 6719-6728.

(21) Pietrzak, M.; Meyerhoff, M. E.; Malinowska, E. Anal. Chim. Acta Polymeric membrane electrodes with improved fluoride selectivity and lifetime based on $\mathrm{Zr}(\mathrm{IV})$ - and $\mathrm{Al}(\mathrm{III})$-tetraphenylporphyrin derivatives. Anal. Chim. Acta 2007, 596, 201-209.

(22) Badr, I. H. A.; Meyerhoff, M. E. Highly selective optical fluoride ion sensor with submicromolar detection limit based on aluminum(III) octaethylporphyrin in thin polymeric film. J. Am. Chem. Soc. 2005, 127, 5318-5319.

(23) Badr, I. H. A.; Meyerhoff, M. E. Highly selective optical fluoride ion sensor with submicromolar detection limit based on aluminum(III) octaethylporphyrin in thin polymeric film. Anal. Chim. Acta 2005, 553, 169-176.

(24) Badr, I. H. A. Potentiometric anion selectivity of polymermembrane electrodes based on cobalt, chromium, and aluminum salens. Anal. Chim. Acta 2006, 570, 176-185.

(25) Meyerhoff, M. E.; Badr, I. H. A. Films for detecting fluoride. U.S. Patent 7,622,075B2, 2009, Pub. No..

(26) Biyareh, M. N.; Rezvani, A. R.; Dashtian, K.; Montazerozohori, M.; Ghaedi, M.; Masoudi Asl, A.; White, J. Potentiometric ionselective electrode based on a new single crystal cadmium(II) schiff base complex for detection of fluoride ion: central composite design optimization. IEEE Sens. J. 2019, 19, 413-425.

(27) Christianson, A. M.; Rivard, E.; Gabbaï, F. P. $1 \lambda^{5}$-Stibaindoles as Lewis acidic, $\pi$-conjugated, fluoride anion responsive platforms. Organometallics 2017, 36, 2670-2676.

(28) Christianson, A. M.; Gabbaï, F. P. A Lewis acidic, $\pi$-conjugated stibaindole with a colorimetric response to anion binding at $\mathrm{Sb}(\mathrm{III})$. Organometallics 2017, 36, 3013-3015.

(29) Kumar, A.; Yang, M.; Kim, M.; Gabbaï, F. P.; Lee, M. H. Offon fluorescence sensing of fluoride by donor-antimony $(\mathrm{V})$ Lewis acids. Organometallics 2017, 36, 4901-4907.

(30) Wade, C. R.; Ke, I.-S.; Gabbaï, F. P. Sensing of aqueous fluoride anions by cationic stibine-palladium complexes. Angew. Chem., Int. Ed. 2012, 51, 478-481.

(31) Bowen, L. H.; Rood, R. T. Solvent extraction of ${ }^{18} \mathrm{~F}$ as tetraphenylstibonium fluoride. J. Inorg. Nucl. Chem. 1966, 28, 19851990.

(32) Hudnall, T. W.; Kim, Y.-M.; Bebbington, M. W. P.; Bourissou, D.; Gabbaï, F. P. Fluoride ion chelation by a bidentate phosphonium/ borane Lewis acid. J. Am. Chem. Soc. 2008, 130, 10890-10891.

(33) Pan, B.; Gabbaï, F. P. $\left[\mathrm{Sb}\left(\mathrm{C}_{6} \mathrm{~F}_{5}\right)_{4}\right]\left[\mathrm{B}\left(\mathrm{C}_{6} \mathrm{~F}_{5}\right)_{4}\right]$ : An air stable, Lewis acidic stibonium salt that activates strong element-fluorine bonds. J. Am. Chem. Soc. 2014, 136, 9564-9567.

(34) Hirai, M.; Gabbaï, F. P. Lewis acidic stiborafluorenes for the fluorescence turn-on sensing of fluoride in drinking water at ppm concentrations. Chem. Sci. 2014, 5, 1886-1893.

(35) Mi, Y.; Bakker, E. Determination of complex formation constants of lipophilic neutral ionophores in solvent polymeric membranes with segmented sandwich membranes. Anal. Chem. 1999, $71,5279-5287$. 
(36) Steinle, E. D.; Schaller, U.; Meyerhoff, M. E. Response characteristics of anion-selective polymer membrane electrodes based on gallium(III), indium(III) and thallium(III) porphyrins. Anal. Sci. 1998, 14, 79-84.

(37) Christianson, A. M.; Gabbaï, F. P. Fluoride and cyanide anion sensing by an $\mathrm{Sb}(\mathrm{V})$-substituted cyclometalated $\mathrm{Ru}$ polypyridyl complex. J. Organomet. Chem. 2017, 847, 154-161.

(38) Christianson, A. M.; Gabbaï, F. P. Anion sensing with a Lewis acidic BODIPY-antimony(V) derivative. Chem. Commun. 2017, 53, 2471-2474. 